\title{
Efeito da adição do copolímero etileno/metacrilato de glicidila (EGMA) nas propriedades reológicas, mecânicas e morfologia do poli (tereftalato de etileno) (PET)
}

\author{
Walber Alexandre do Nascimento ${ }^{1 *}$ (1) \\ Henriqueta Portilho da Silva \\ Pankaj Agrawal ${ }^{2}$ \\ Tomás Jeferson Alves de Mélo ${ }^{2}$
}

\section{Resumo}

O intuito deste trabalho foi estudar o efeito EGMA nas propriedades reológicas, mecânicas e na morfologia do PET. Diversas composições formuladas com o EGMA e a matriz de PET foram produzidas. Utilizaram-se reometria de torque, FTIR, ensaios mecânicos e MEV para caracterizar as blendas. Os resultados obtidos por reometria de torque indicaram um aumento da amplitude das curvas de torque, e consequentemente da viscosidade do fundido, com o aumento da concentração de EGMA. O FTIR indicou reações ocorridas entre os grupos funcionais do EGMA, com os grupos terminais do PET, sendo esse o fator preponderante para que houvesse aumento do torque na análise por reometria. A propriedade mecânica de RI teve um aumento de quase 8x, quando comparado ao PET puro, apresentando também uma leve redução da rigidez e da RT com o aumento da concentração de EGMA. As análises morfológicas indicaram a formação de uma estrutura miscível/compatível, ou seja, sem evidente separação de fase, o que pode ter corroborado na obtenção da tenacificação do PET como observado também no aumento crescente da RI.

Palavras-chave: PET; EGMA; Blendas; RI.

\section{Effect of adding the ethylene/glycidyl methacrylate (EGMA) copolymer on the rheological and mechanical properties, and morphology of poly (ethylene terephthalate) (PET)}

\begin{abstract}
This work aimed to study the effect of EGMA on the rheological, mechanical, and morphological properties of PET. Several compositions formulated with EGMA and the PET matrix were produced. Torque rheometry, FTIR, mechanical tests, and SEM were used to characterize the blends. The results obtained by torque rheometry indicated an increase in the amplitude of the torque curves, and consequently in the melt viscosity, with the increase in the concentration of EGMA. The FTIR indicated reactions that occurred between the functional groups of EGMA, with the terminal groups of PET, which is the main factor for an increase in torque in the analysis by rheometry. The mechanical property of IS increased almost $8 \mathrm{x}$ when compared to pure PET, also showing a slight reduction in stiffness and TS with an increase in the concentration of EGMA. Morphological analyzes indicated the formation of a miscible/compatible structure, that is, without evident phase separation, which may have corroborated in obtaining PET toughening, as also observed in the progressive increase in IS.
\end{abstract}

Keywords: PET; EGMA; Blends; IS.

${ }^{1}$ Faculdade de Engenharia Mecânica, Universidade Federal do Pará - UFPA, Tucuruí, PA, Brasil.

${ }^{2}$ Departamento de Engenharia de Materiais, Universidade Federal de Campina Grande - UFCG, Campina Grande, PB, Brasil.

*Autor correspondente: wan@ufpa.br

2176-1523 (c) 2021. Nascimento et al. Publicado pela ABM. Este é um artigo publicado em acesso aberto (Open Access) sob a licença Creative Commons Attribution, que permite uso, distribuição e reprodução em qualquer meio, sem restrições desde que o trabalho original seja corretamente citado. 


\section{Introdução}

O PET é um dos polímeros amplamente utilizado no mundo nas mais variadas aplicações. É um poliéster termoplástico de grande interesse comercial devido as suas propriedades químicas (barreira a gases, principalmente $\mathrm{CO}_{2}$ ), óticas (transparência) e mecânicas (rigidez) peculiares. É usado, principalmente, na produção de fios têxteis e embalagens para bebidas carbonatadas e não-carbonatadas $[1,2]$.

Uma das dificuldades de se utilizar o PET está relacionado ao seu processamento. Uma das técnicas utilizadas para melhorar o seu processamento está na formação de blendas com o uso de copolímeros que sejam miscíveis e possuam interações com o PET. Esta melhoria está associada ao aumento da viscosidade, pois o PET, quando processado isoladamente, pode apresentar vários processos degradativos como, por exemplo, a termooxidação e hidrólise. Isso reduz drasticamente essa propriedade reológica (viscosidade), dificultando um fluxo contínuo da massa polimérica fundida no equipamento de processamento [3]. A redução da viscosidade causa também a redução das propriedades mecânicas após moldagem, principalmente rigidez e tenacidade $[4,5]$.

$\mathrm{Na}$ literatura observa-se que estudos relacionados à reatividade de copolímeros com poliésteres, como o PET, que apresentam grupos terminais hidroxila $(-\mathrm{OH})$ e carboxila $(-\mathrm{COOH})$ em sua estrutura química, foram realizados visando modificar as propriedades de fluxo, quando em processamento e também as propriedades mecânicas (módulo) e tenacidade, após a moldagem por processos convencionais como extrusão e injeção [6-8]. Alguns autores identificaram que os copolímeros contendo grupos funcionais metacrilato de glicidila (-GMA) em sua composição química, reagem com poliésteres durante o processamento, atuando como agentes de extensão e/ ou ramificação da cadeia aumentando a viscosidade do fundido e consequentemente melhorando as propriedades mecânicas, principalmente resistência ao impacto, sem perder de forma significativa a rigidez [9]. Veremos agora uma alguns desses trabalhos.

Xanthos et al. [10] estudaram a reatividade do $\{\mathrm{N}$, N'-bis [3 (carbo-2', 3-epoxi propoxi) fenil] piremelitimida\} diepóxido e do copolímero EGMA com os grupos terminais -COOH e-OH do PET. Os autores concluíram que o diepóxido e o EGMA reagiram com PET, atuando como um extensor de cadeia e melhorando o fluxo da massa polimérica no processamento.

Chapleau e Huneault [11] investigaram o efeito de copolímeros contendo -GMA na resistência a tração, módulo de elasticidade (rigidez), alongamento na ruptura e resistência ao impacto (tenacidade) e morfologia do PET. Eles observaram que a adição dos copolímeros ao PET diminuiu a rigidez e a resistência à tração na ruptura, enquanto o alongamento na ruptura e a tenacidade aumentaram. Eles verificaram por MEV que a presença desses copolímeros favoreceu o mecanismo de fratura dúctil no ensaio de resistência ao impacto, o que explica o aumento da tenacidade em detrimento da rigidez.

Tan et al. [12] investigaram o efeito do copolímero etileno-propileno-dieno enxertado com metacrilato de glicidila (EPDMgGMA) nas propriedades do PET. Os autores observaram que a resistência ao impacto do PET aumentou substancialmente com a adição desse copolímero e foi mais pronunciado para a mistura contendo $30 \%$ de copolímero de EPDMgGMA. Os autores concluíram ainda que o-GMA é um extensor de cadeia eficaz para PET, melhorando o seu processamento e atuando também como eficiente agente tenacificante.

Nechifor et al. [13] realizaram um estudo de revisão sobre o efeito de vários compatibilizantes, principalmente em polímeros mais reativos, como o PET. Os autores concluíram que o metacrilato de glicidila (-GMA), que está também presente no EGMA, é um reagente eficiente devido a sua boa reatividade com os ácidos carboxílicos de poliésteres, como o PET, e grupos amino de poliamidas.

Araújo et al. [14] ainda verificaram o efeito de copolímeros contendo ácido acrílico (-AA) e -GMA nas propriedades reológicas e morfologia do poliéster poliácido lático (PLA). Os autores verificaram que o efeito da adição de copolímeros contendo -GMA melhoraram o processamento do PLA além de quase triplicar os valores de RI para as blendas formuladas.

Chen et al. [15] estudaram o efeito da adição do polímero PGMA, que contêm o grupo reativo -GMA, no comportamento térmico, mecânico e reológico do PET reciclado. Os autores observaram não só um aumento na rigidez com o aumento da temperatura de transição vítrea $\left(\mathrm{T}_{\mathrm{g}}\right)$, provocado pela presença do PGMA, mas também verificaram que as blendas formuladas aumentaram a viscosidade do fundido no processamento, favorecendo o seu uso em aplicações na reciclagem de PET como embalagens, fios ecológicos e tecidos não tecidos (TNT).

De acordo com o exposto observamos a importância do estudo do efeito da adição do copolímeros que contêm -GMA, como o EGMA, no efeito das propriedades reológicas, mecânicas e morfologia do PET. Para isso foram formuladas várias blendas para caracterizar a influência desse copolímero na matriz de PET, como: reometria de torque (no estado fundido); ensaios de tração e impacto; FTIR e MEV.

\section{Materiais e métodos}

\subsection{Materiais}

- Poli (tereftalato de etileno) (PET) da M \& G (Cleartuf ${ }^{\circledR}$ Turbo $^{\mathrm{TM}}$ ) com Viscosidade Intrínseca-VI (3J-Lab-050) $=0,80 \pm 0,02 \mathrm{dl} / \mathrm{g}$ e ponto de fusão $\mathrm{PF}(\mathrm{M} \& \mathrm{G} / \mathrm{QC}-06)=246^{\circ} \mathrm{C}$. A estrutura química do polímero está apresentada na Tabela 1 ; 
- Copolímero de etileno-metacrilato de glicidila (EGMA), contendo $8 \%$ de metacrilato de glicidila, Lotader ${ }^{\circledR}$ AX 8840, com índice de fluidez (ASTM $\mathrm{D} 1238)=5 \mathrm{~g} / 10 \mathrm{~min}$, densidade $($ ASTM D $792)=0,94 \mathrm{~g} / \mathrm{cm} 3$, ponto de fusão $-\mathrm{PF}(\mathrm{DSC})=106^{\circ} \mathrm{C}$, módulo $($ ASTM D790) $=85 \mathrm{MPa}$, alongamento na ruptura $($ ASTM D638) $=400 \%$, resistência a tração - RT $($ ASTM D638) = $8 \mathrm{MPa}$. A estrutura química do copolímero está apresentada na Tabela 1.

\subsection{Métodos}

\subsubsection{Reometria de torque}

A análise reológica do PET e das blendas foi realizada por reometria de torque utilizando o equipamento Haake Polylab QC Rheomix 600 da Thermo Scientific, equipado com rotores do tipo roller, sob atmosfera de ar, utilizando velocidade de $50 \mathrm{rpm}$ e temperatura nominal de $260^{\circ} \mathrm{C}$ durante 10 minutos. Foi utilizada uma massa total de 60 g. Antes das análises por reometria de torque, o PET foi seco em estufa a vácuo a $110^{\circ} \mathrm{C} / 24$ horas enquanto que o EGMA foi utilizado como recebido. Foram realizadas composições com a finalidade de avaliar o comportamento reológico da blenda PET/EGMA quando se aumenta a quantidade desse copolímero. A escolha foi definida de forma a se ter baixas $(5 \%)$ até médias concentrações $(20 \%)$. Os teores (\% peso) das blendas estão apresentadas na Tabela 2.

\subsubsection{Processamento por extrusão}

As blendas de PET/EGMA foram preparadas em extrusora dupla rosca modular corrotacional Coperion Werner \& Pfleiderer ZSK $18 \mathrm{~mm}(\mathrm{~L} / \mathrm{D}=40)$ com uma velocidade de $250 \mathrm{rpm}$ e taxa de alimentação de $3 \mathrm{~kg} /$ hora. O perfil térmico de aquecimento da extrusora até o cabeçote foi mantido em $280^{\circ} \mathrm{C}$ em todas as zonas. O PET foi seco nas mesmas condições do item 2.1.1 antes do processamento por extrusão. O copolímero EGMA foi utilizado como recebido, ou seja, não passou por uma secagem antes de ser incorporado junto ao PET. Essas condições foram utilizadas devido a obtenção do melhor perfil de extrudado contínuo e sem defeitos durante os testes de extrusão. A configuração da rosca utilizada foi composta com elementos de transporte, distributivos e dispersivos para obtenção de um melhor processo de mistura das blendas (Figura 1). Foram elaboradas as mesmas composições da Tabela 2.

Todas as composições da Tabela 2, após o processamento por extrusão, foram granuladas e secas novamente em estufa a $110^{\circ} \mathrm{C} / 24$ horas. Os corpos de prova foram moldados em uma injetora Fluidmec H30/40180 da ARBURG, em uma temperatura de processamento de $300^{\circ} \mathrm{C}$ com a temperatura do molde de $20^{\circ} \mathrm{C}$.

\subsubsection{Ensaios de tração e impacto}

Os ensaios de tração foram realizados em uma máquina de ensaio universal Shimadzu AG-IS $100 \mathrm{kN}$, de acordo com a norma ASTM-D 638, em amostras de tipo I, operando a uma taxa de deformação de $50 \mathrm{~mm} /$ minuto. Os testes de resistência ao impacto Izod foram realizados de acordo com a norma ASTM-D $256 \mathrm{em}$ amostras, entalhadas, com dimensões de $63,5 \mathrm{~mm} \mathrm{x}$ $12,7 \mathrm{~mm} \times 33,2 \mathrm{~mm}$, à temperatura ambiente usando um aparelho

Tabela 1. Estrutura química do PET e do copolímero EGMA

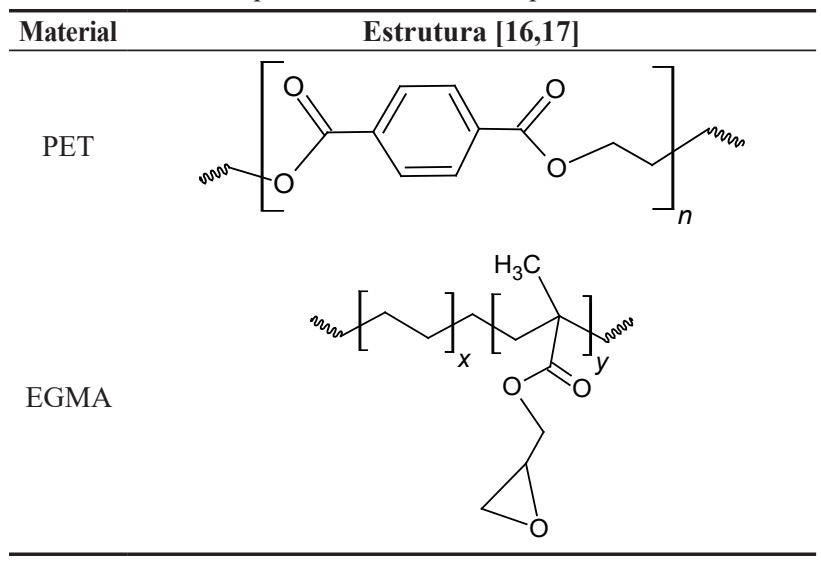

Tabela 2. Composições das blendas

\begin{tabular}{ccc}
\hline $\begin{array}{c}\text { Nomenclatura das } \\
\text { Blendas }\end{array}$ & PET (\%)* & EGMA (\%)* \\
\hline EGMA 5 & 95 & 5 \\
EGMA 10 & 90 & 10 \\
EGMA 15 & 85 & 15 \\
EGMA 20 & 80 & 20 \\
\hline
\end{tabular}

*Porcentagem em peso.

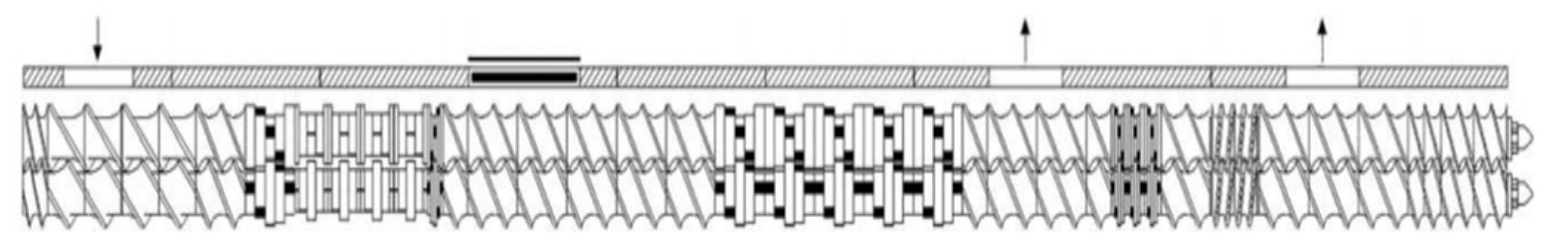

Figura 1. Configuração da rosca utilizada na formação das blendas. 
Ceast Resil 5.5. Foram utilizados 7 corpos de prova em média para realização dos ensaios em cada composição da Tabela 2.

\subsubsection{Espectroscopia na região do infravermelho por transformada de fourier (FTIR)}

As análises por espectroscopia na região do infravermelho por transformada de Fourier (FTIR), foram realizadas em um espectrômetro de infravermelho de marca Perkin Elmer, modelo Spectrum 400 FT Mid-IR, operando no modo de reflexão total atenuada (ATR). As amostras foram caracterizadas em forma de pequenas lascas raspadas dos corpos de prova injetados. Esta técnica foi utilizada para avaliar as possíveis interações entre os grupos reativos do copolímero com os do PET.

\subsubsection{Microscopia eletrônica de varredura (MEV)}

Para a análise morfológica as amostras, provenientes da superfície de fratura dos corpos de prova do ensaio de

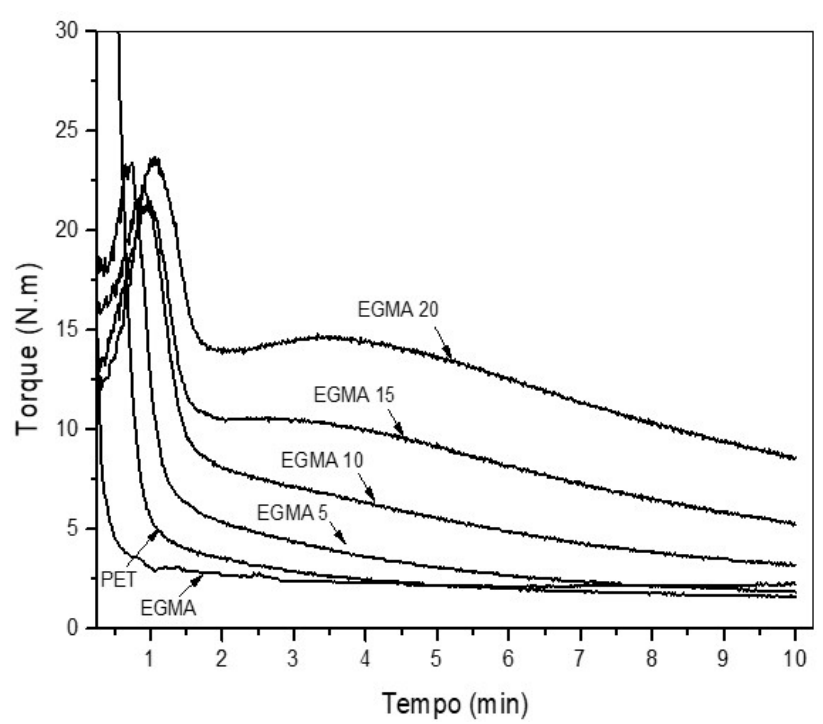

Figura 2. Curvas torque em função do tempo para o PET, EGMA e para as blendas PET/EGMA. resistência ao impacto, foram revestidas com ouro para então serem analisadas por Microscopia Eletrônica de Varredura (MEV) utilizando um microscópio eletrônico de varredura Shimadzu SS X550 Super Scan. A voltagem usada no filamento foi de $15 \mathrm{kV}$. Esta técnica foi utilizada para avaliar a morfologia das blendas e correlacionar com as propriedades mecânicas.

\section{Resultados e discussão}

\subsection{Análise reológica por reometria de torque}

A Figura 2 ilustra as curvas de torque em função do tempo do PET e das blendas de PET com EGMA. O PET, após o carregamento no estado sólido dentro da câmara de mistura, apresenta um pico máximo de torque em torno de 110 N.m (não mostrado no gráfico) e, depois de sua fusão, ocorre uma redução acentuada do torque até o tempo de 1 minuto e mais lentamente até o tempo final do processo em 10 minutos. Em relação às blendas de PET com EGMA, observam-se mudanças no comportamento das curvas de torque, há redução de intensidade e deslocamento no tempo do pico máximo do PET presente nas blendas, provavelmente os copolímeros que têm pontos de fusão inferior ao PET, fundiram antes e promoveram um efeito plastificante nos grânulos do PET, retardando sua fusão em aproximadamente 1 minuto. Após este tempo, observa-se que as curvas de torque das blendas deslocam-se para cima quanto maior for à concentração dos copolímeros. Este efeito é mais pronunciado para a blendas PET/EGMA que apresenta concentração mais elevada de 15 e $20 \%$.

O aumento do torque após a fusão dos componentes da blenda pode indicar ocorrência de reação química entre ambos. De acordo com Pracella et al. [17], as reações químicas entre os grupos terminais hidroxila (-OH) e carboxila $(-\mathrm{COOH})$ do PET com grupos epóxis, formam grupos éter e éster (Figura 3), respectivamente e, por conseguinte, promovem
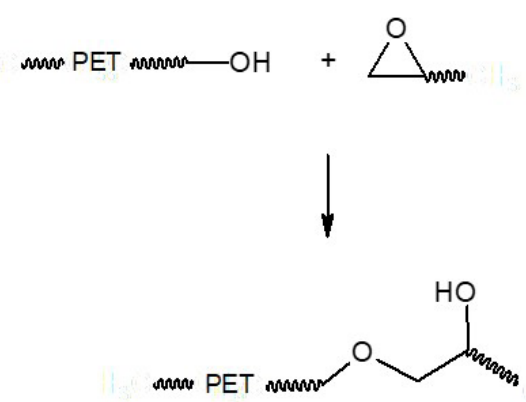

b)

Figura 3. Reações dos grupos terminais carboxila e álcool do PET com o anel epóxi do GMA [18,19]. (a) Reação de esterificação; (b) Reação de eterificação. 
aumento de massa molecular por meio de extensão de cadeia e/ou ramificações que proporcionam aumento de viscosidade e elasticidade do PET, consequentemente aumento do torque durante o processamento.

De uma maneira geral, por meio da reometria de torque, verifica-se que os grupos epóxi do EGMA apresentam reatividade com os grupos terminais - $\mathrm{OH}$ e - $\mathrm{COOH}$ do $\mathrm{PET}$, corroborado pelo aumento da viscosidade e, por conseguinte, do torque.

Verifica-se ainda que, por mais que o copolímero EGMA favoreça o aumento da viscosidade do fundido em concentrações crescentes, os possíveis efeitos da cisão de cadeia polimérica, promovida pelos processo de degradação hidrolítica, termo-oxidativa e termomecânica, torna-se mais efetivo após 1 minuto de processamento com consequente redução da curva torque $\mathrm{x}$ tempo $[2,20-22]$.

\subsection{Espectroscopia na região do infravermelho por transformada de Fourier (FTIR)}

Os espectros de FTIR do PET, EGMA e das blendas PET/EGMA são ilustrados na Figura 4. Observa-se a existência de bandas típicas presentes na cadeia do PET, situadas em 1000 e $800 \mathrm{~cm}^{-1}$, que representam a vibração de deformação do grupo éster (-COO-) [23]. No espectro do EGMA, verifica-se a presença do anel epóxi presente nos grupos metacrilato de glicidila (GMA) caracterizada pela existência de uma banda localizada entre $908-912 \mathrm{~cm}^{-1}$, correspondente à contração das ligações $\mathrm{C}-\mathrm{C}$, e estiramento das ligações $\mathrm{C}-\mathrm{O}[7,17]$. A banda do anel epóxi pode ser visualizada na Figura 4 para o EGMA indicada por uma seta no espectro dos copolímeros a $910 \mathrm{~cm}^{-1}$. É possível observar o desaparecimento desta banda nas blendas, o que sugere a ocorrência de reação entre anel epóxi presente no copolímero em todas as composições e os grupos terminais

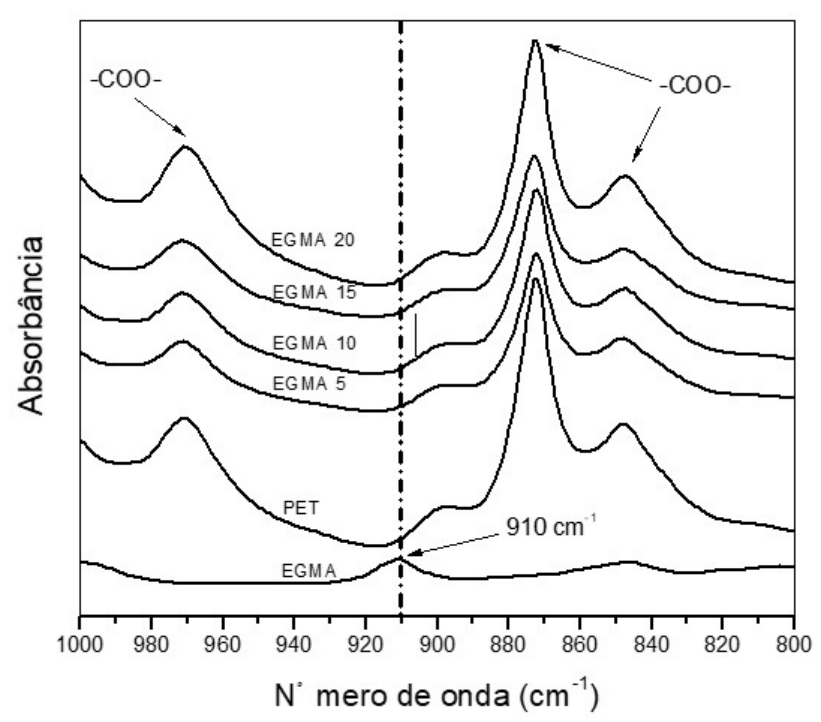

Figura 4. Espectros de FTIR para o PET, EGMA e para as blendas PET/EGMA. carboxilas e/ou hidroxilas do PET (ver Figuras 3). Portanto, os resultados de FTIR corroboram as mudanças observadas nas análises reológicas.

\subsection{Propriedades mecânicas}

Os valores de módulo de elasticidade (E), resistência à tração (RT) e resistência ao impacto (RI), obtidos a partir dos ensaios mecânicos realizados para o PET e para as blendas PET/EGMA, são apresentados na Tabela 3.

Observa-se que a adição de quantidades crescentes do copolímero diminui o módulo elástico e a resistência à tração e aumenta a resistência ao impacto. Isto indica que para as blendas de PET/EGMA, há redução da rigidez e aumento na tenacidade, promovidos pelos copolímeros que têm baixa rigidez e elevada tenacidade, conforme os dados do fabricante descritos no item 2.1.

Estes resultados estão em consonância com Silveira [23], Baouz et al. [24] e Feng [25] quando eles estudaram o efeito de copolímeros contendo -GMA numa blenda com poliácido láctico (PLA) como matriz. Analogamente Brito et al. [22,26] observaram melhora significativa na resistência ao impacto de blendas de PLA/ EMAGMA. Os autores verificaram que copolímeros com grupos -GMA são efetivos agentes compatibilizantes para poliésteres como o PLA, PET, PBT, etc. devido a esse polímeros apresentarem grupos reativos hidroxila $(-\mathrm{OH})$ e carboxila (-COOH) que reagem prontamente com o anel epóxi do -GMA, favorecendo a redução da tensão interfacial e melhorando a compatibilidade de polímeros imiscíveis com consequente avanço nas propriedades mecânicas como tenacidade e resistência ao impacto com relativa redução da rigidez da blenda formada. Outros autores como Yuryev et al. [27] e Benhamida et al. [28] utilizaram etilenoacrilato de butila-metacrilato de glicidila (EBAGMA) para estudar o seu efeito em blendas PET/EBAGMA e PLA/ EBAGMA. Os autores verificaram aumento crescente das propriedades mecânicas, inerentes a resistência ao impacto, tenacidade e alongamento na ruptura com relativo decréscimo da rigidez, com o uso desse copolímero. De uma maneira geral, o uso de copolímeros a base de -GMA são efetivos compatibilizantes para melhorar a tenacidade, resistência ao impacto e alongamento a ruptura de poliésteres como o PET, PLA, PBT etc.

Tabela 3. Propriedades mecânicas do PET e das blendas PET/EGMA

\begin{tabular}{cccr}
\hline Amostra & E $(\mathbf{G P a})$ & RT $(\mathbf{M P a})$ & \multicolumn{1}{c}{ RI $\mathbf{( J / m )}$} \\
\hline PET & $2,29 \pm 0,04$ & $56,90 \pm 0,49$ & $32,10 \pm 2,84$ \\
EGMA5 & $2,07 \pm 0,03$ & $47,83 \pm 0,53$ & $55,31 \pm 1,31$ \\
EGMA10 & $1,84 \pm 0,03$ & $46,03 \pm 0,74$ & $76,45 \pm 1,64$ \\
EGMA15 & $1,56 \pm 0,02$ & $41,31 \pm 0,17$ & $172,47 \pm 6,47$ \\
EGMA20 & $1,36 \pm 0,04$ & $39,24 \pm 0,19$ & $240,35 \pm 7,55$ \\
\hline
\end{tabular}

$\mathrm{E}=$ módulo de elasticidade; $\mathrm{RT}=$ resistência à tração; $\mathrm{RI}=$ resistência ao impacto. 


\subsection{Microscopia eletrônica de varredura (MEV)}

A Figura 5 apresenta as micrografias do PET (Figura 5a) e das blendas PET/EGMA (Figuras 5b, c, d e e). Observamos na Figura 5a uma fratura frágil do PET, ou seja, uma superfície de fratura mais lisa nas condições do ensaio de impacto. Na Figura 5b, quando se adiciona o EGMA, a superfície de fratura da amostra começa a se tornar rugosa, ou seja, apresenta fratura dúctil em sua superfície. Esse fato fica mais pronunciado nas Figuras $5 \mathrm{c}, 5 \mathrm{~d}$ e $5 \mathrm{e}$ com o aumento da concentração do EGMA. A ductilidade/ tenacidade das blendas é corroborada pelas propriedades mecânicas apresentadas na Tabela 3 com o aumento progressivo da RI. Outro fato importante identificado é o não aparecimento de domínios da fase dispersa de EGMA na forma de "gotas", como apresentado por outros trabalhos como os de Baouz et al. [24], Feng et al. [25], Yuryev et al. [27] e Benhamida et al. [28]. Os autores desses trabalhos verificaram que a fase dispersa de copolímeros contendo o grupo -GMA apresentaram baixa tensão interfacial na matriz de poliésteres, favorecendo o processo de adesão. Fica evidenciado que o EGMA apresenta uma boa interação com a matriz de PET, o que reduz a tensão interfacial entre as fases facilitando a incorporação da fase dispersa sem haver segregação das fases. Resultado semelhante foi relatado por Chapleau e Huneault [11] que investigaram o efeito de modificadores não reativos e reativos nas propriedades de impacto do PET. Os autores observaram que o tamanho dos domínios da fase dispersa permaneceu quase constante com o aumento da concentração do modificador reativo (contendo grupo -GMA).
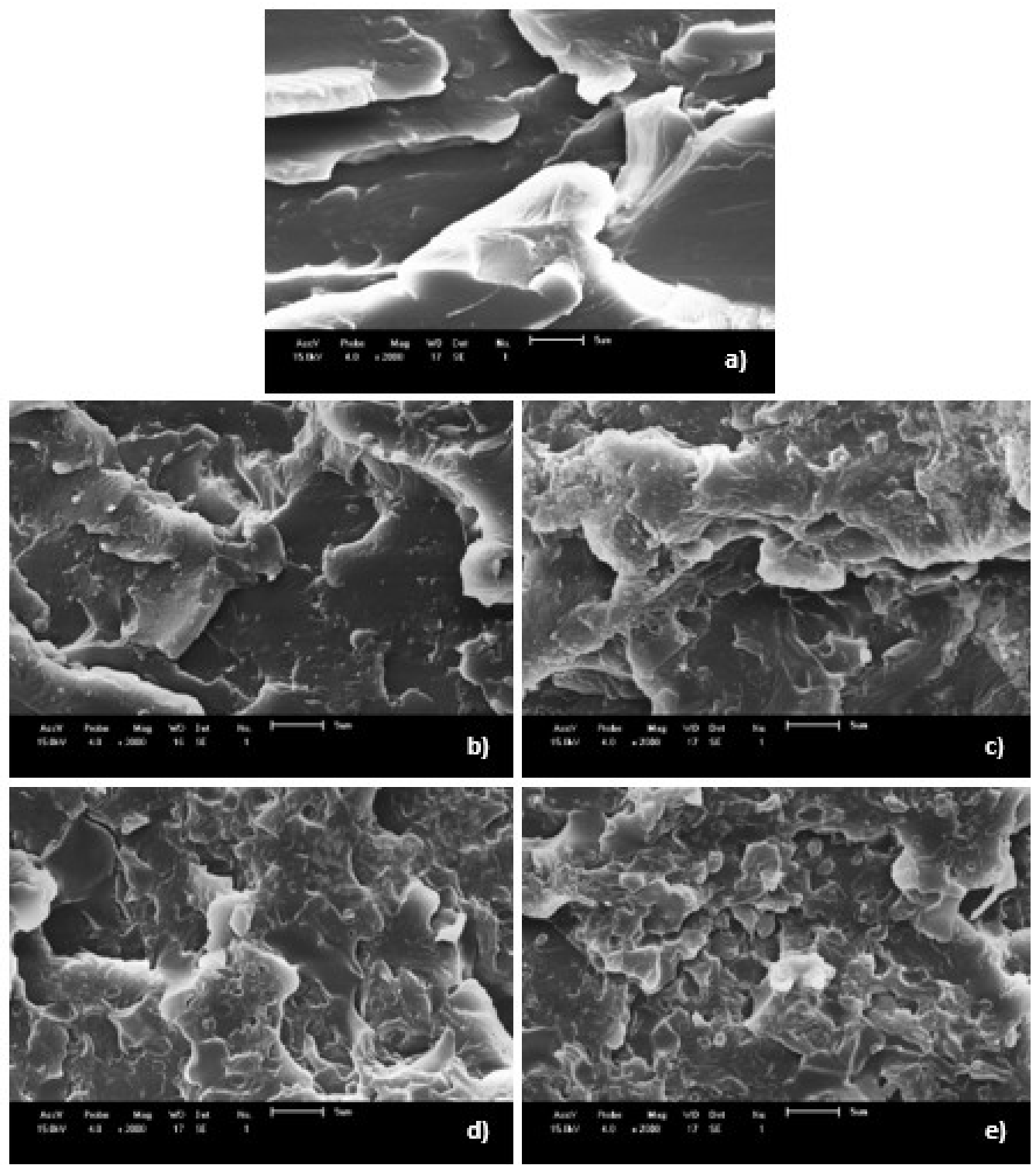

Figura 5. Fotomicrografias do (a) PET; (b) EGMA 5; (c) EGMA 10; (d) EGMA 15; (e) EGMA 20. Aumento de $2000 \mathrm{X}$ e escala de 5 $\mu$ m. 
Efeito da adição do copolímero etileno/metacrilato de glicidila (EGMA) nas propriedades reológicas, mecânicas e morfologia do poli (tereftalato de etileno) (PET)

Eles atribuíram esse resultado às reações químicas entre os grupos funcionais de PET e os grupos funcionais presentes no modificador formando um copolímero na interface e suprimindo a coalescência entre as partículas modificadoras.

\section{Conclusão}

Neste trabalho foi investigado o efeito da adição do copolímero EGMA nas propriedades reológicas (reometria de torque), mecânicas (tração e impacto), e morfologia (MEV) na matriz de PET. As análises reológicas indicaram que houve mudanças no comportamento do PET devido às reações ocorridas entre os grupos funcionais metacrilatos de glicidila, presentes no copolímero com os grupos terminais carboxilas e hidroxilas do PET. Estas reações foram corroboradas por FTIR.
As reações entre os componentes das blendas modificaram as propriedades mecânicas do PET. Praticamente não observamos domínios da fase dispersa de EGMA no formato de "gotas" com um aumento de 2000x, indicando adesão interfacial com a matriz de PET. As análises morfológicas indicaram uma correlação direta com as propriedades mecânicas onde obteve-se uma resistência ao impacto de até oito vezes superior ao do PET puro indicando que a matriz de PET foi tenacificada pela adição do desse copolímero.

\section{Agradecimentos}

Os autores agradecem a UFPA, UFCG e CAPES pelo apoio durante a pesquisa e as empresas Gigaplast Representação \& Consultoria e a Arkema pela doação do PET e do EGMA, respectivamente.

\section{Referências}

1 Associação Brasileira da Indústria do PET [página da internet]. São Paulo: ABIPET; 2017 [acesso em 13 abr. 2020]. Disponível em: http://www.abipet.org.br

2 Romão W, Spinacé MAS, Paoli M-AD. Poli(Tereftalato de Etileno), PET: uma revisão sobre os processos de síntese, mecanismos de degradação e sua reciclagem. Polímeros: Ciência e Tecnologia. 2009;19(2):121-132.

3 Carté TL, Moet A. Morphological origin of super toughness in poly (ethylene terephthalate)/polyethylene blends. Journal of Applied Polymer Science. 1993;48(4):611-624.

4 Dimitrova TL, La Mantia FP, Pilati F, Toselli M, Valenza A, Visco A. On the compatibilization of PET/HDPE blends through a new class of copolyesters. Polymer. 2000;41(13):4817-4824.

5 Iñiguez CG, Michel E, González-Romero VM, González-Nuñez R. Morphological stability of postconsumer PET/ HDPE blends. Polymer Bulletin. 2000;45(3):295-302.

6 Pereira LM, Corrêa AC, Souza MM Fo, Rosa MF, Ito EN. Rheological, morphological and mechanical characterization of recycled poly (ethylene terephthalate) blends and composites. Materials Research. 2017;20(3):791-800.

7 Meyva Y, Kaynak C. Influences of three different ethylene copolymers on the toughness and other properties of polylactide. Plastics, Rubber and Composites. 2016;45(5):189-198.

8 Mascia L, Haworth B, Vignali A, Megna R, Acierno D, Russo P. Thermal transitions and solidification kinetics of poly(lactic acid) and blends with epoxidized natural rubber. Thermochimica Acta. 2016;633(1):82-90.

9 Gurunathan T, Chung JS, Nayak SK. Reactive compatibilization of biobased polyurethane prepolymer toughening polylactide prepared by melt blending. Journal of Polymers and the Environment. 2016;24(4):287-297.

10 Xanthos M, Young M-W, Karayanndis GP, Bikiaris DN. Reactive modification of polyethyiene terephthaiate with polyepoxides. Polymer Engineering and Science. 2001;41(4):643-655.

11 Chapleau N, Huneault MA. Impact modification of poly(ethylene terephthalate). Journal of Applied Polymer Science. 2003;90(11):2919-2932.

12 Tan Z, Liu S, Cui X, Sun S, Zhang H. Application of macromolecular chain extender and contribution to the toughening of poly(ethylene terephthalate). Journal of Thermoplastic Composite Materials. 2016;29(6):833-849.

13 Nechifor M, Tanasă F, Teacă C-A, Zănoagă M. Compatibilization strategies toward new polymer materials from re-/ up-cycled plastics. International Journal of Polymer Analysis and Characterization. 2018;23(8):740-757.

14 Araújo JP, Oliveira ADB, Cavalcanti SN, Agrawal P, Melo TJA. Combined effect of copolymers and of the mixing sequence on the rheological properties and morphology of poly(lactic acid) matrix blends. Materials Chemistry and Physics. 2019;237:121818. 
15 Chen C-W, Liu P-H, Lin F-J, Cho C-J, Wang L-Y, Mao H-I, et al. Influence of different molecular weights and concentrations of poly(glycidyl methacrylate) on recycled poly(ethylene terephthalate): a thermal, mechanical, and rheological study. Journal of Polymers and the Environment. 2020;28(11):2880-2892.

16 Silveira EBD. Estudo da tenacificação do PLA pela adição de elastômero termoplástico EMAGMA [dissertação]. São Paulo: Escola Politécnica, Universidade de São Paulo; 2016.

17 Pracella M, Chionna D, Pawlak A, Galeski A. Reactive mixing of PET and PET/PP blends with glycidyl methacrylate-modified styrene-b-(ethylene-co-olefin) block copolymers. Journal of Applied Polymer Science. 2005;98(5):2201-2211.

18 Visakh PM, Liang M. Poly(ethylene terephtalate) based blends, composites and nanocomposites. Oxford: Elsevier; 2015.

19 Venkatachalam S, Nayak SG, Labde JV, Gharal PR, Rao K, Kelkar AK. Degradation and recyclability of poly (ethylene terephthalate). London: IntechOpen; 2012 [acesso em 13 abr. 2020]. Disponível em: https://www. intechopen.com/books/polyester/degradation-and-recyclability-of-poly-ethylene-terephthalate-

20 Nayak S, Labde J, Geedh S, Jaisingh SK, Rao K, Venkatachalam S, et al. Study on degradation reactions in polyethylene terephthalate containing 5-sulpho isophthalyl moieties. Journal of Applied Polymer Science. 2010;118(5):2791-2800.

21 Awaja F, Pavel D. Recycling of PET. European Polymer Journal. 2005;41(7):1453-1477.

22 Brito GF, Agrawal P, Araújo EM, Melo TJA. Tenacificação do poli(ácido lático pela adição do terpolímero (etileno/ acrilato de etila/metacrilato de glicidila). Polímeros. 2012;22(2):164.

23 Silveira EB. Estudo da tenacificação do PLA pela adição de elastômero termoplástico EMAGMA [dissertação]. São Paulo: Universidade de São Paulo; 2016.

24 Baouz T, Rezgui F, Yilmazer U. Ethylene-methyl acrylate-glycidyl methacrylate toughened poly(lactic acid) nanocomposites. Journal of Applied Polymer Science. 2013;128(5):3193-3204.

25 Feng Y, Zhao G, Yin J, Jiang W. Reactive compatibilization of high-impact poly(lactic acid)/ethylene copolymer blends catalyzed by N,N-dimethylstearylamine. Polymer International. 2014;63(7):1263-1269.

26 Brito GF, Agrawal P, Mélo TJA. Mechanical and morphological properties of PLA/BioPE blend compatibilized with E-GMA and EMA-GMA copolymers. Macromolecular Symposia. 2016;367(1):176-182.

27 Yuryev Y, Mohanty AK, Misra M. A new approach to supertough poly(lactic acid): a high temperature reactive blending. Macromolecular Materials and Engineering. 2016;301(12):1443-1453.

28 Benhamida A, Kaci M, Cimmino S, Silvestre C, Duraccio D. Evaluation of the effectiveness of new compatibilizers based on EBAGMA-LDPE and EBAGMA-PET masterbatches for LDPE/PET blends. Macromolecular Materials and Engineering. 2010;295(3):222-232.

Recebido em: 30 Abr. 2020

Aceito em: 30 Out. 2020 